\title{
Temperature measurement by thermocouple probe: influences of variable fluid velocity on the dynamic characteristics of thermocouple
}

\author{
Zhaoxin Yang ${ }^{1, a}$, Xiaofeng Meng ${ }^{1, b}$
}

${ }^{1}$ Science and Technology on Inertial Laboratory, Beijing University of Aeronautics and Astronautics, Beijing, 100191, China

a tutu198210@gmail.com, b mengxf@buaa.edu.cn

Keywords: Thermocouple, Dynamic measurement, time constant, fluid velocity

Abstract. The problem of dynamic thermocouple compensation is considered. Taking into account the nonlinear dependency of the thermocouple time constant on fluid velocity, a thermocouple model and a time constant model are proposed successively for estimation of nonlinear thermocouple time constant. This technique is most suitable for measuring dynamic temperature because of the precise description of time constant. The improve scheme offers better compensation of the thermocouple response. The parameter estimation is performed using GTLS to cope with the noise present on the regressors and the output.

\section{Instruction}

In many industrial applications, thermocouples are used due to their high credibility and good linear dependence with temperature. However there are drawbacks of thermocouples, the most important of which is inaccuracy of measuring high frequency temperature fluctuations [1]. So, by establishment of the dynamic model, thermocouple input can be reconstructed. In general, thermocouple was model as a first order nonlinear model. Time constant is the undetermined parameter of the model. As the present research, a fixed (mean) time constant [2] is almost always used, although it is variable in fluctuating velocity and thermal field. The simplification of the procedure of modeling and data processing is based on the cost of measurement accuracy.

In this paper, we propose a method which identifies the system of thermocouple denoted as $\mathrm{G}_{\text {thermo }}(\tau)$ whose input and output signal is fluid temperature and thermocouple response, respectively, and that of time constant denote as $\mathrm{G}_{\mathrm{v}-\tau}(\mathrm{u})$ whose input and output signal is fluid velocity and time constant of thermocouple, respectively, can further improve the accuracy of dynamic compensation of thermocouple. As shown in Fig. $1, \mathrm{G}_{\text {thermo }}(\tau)$ and $\mathrm{G}_{\mathrm{v}-\tau}(\mathrm{u})$ are both excited by step signal, meanwhile their outputs are corrupted by noise $\eta_{1}$ and $\eta_{2}$ respectively, and $\tau_{\mathrm{S}}$ is sampling interval. We can obtain the relationship between time constant and fluid velocity when the model of $\mathrm{G}_{\text {thermo }}(\tau)$ and $\mathrm{G}_{\mathrm{v}-\tau}(\mathrm{u})$ is established.

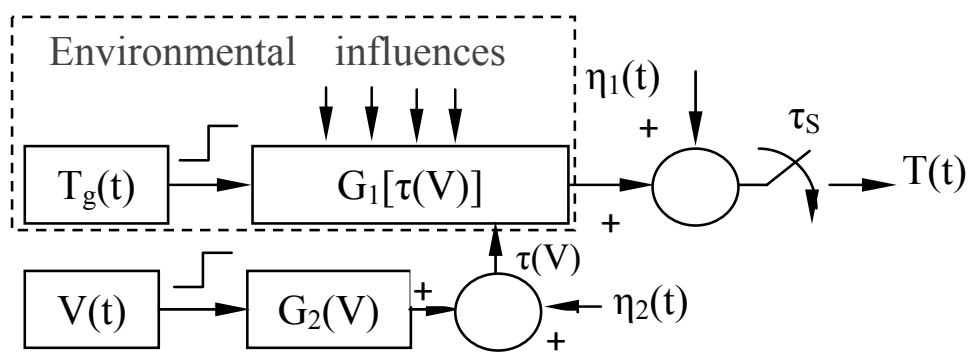

Fig. 1. Block diagram of simulated thermocouple measurement system

\section{Calibration System}

It is a water bath temperature calibration system, shown in Fig. 2. Thermocouple fixed in the mounting block connected with the slide bar. Lock the slide bar by the lock pin when pull it up to the cross beam. First release the lock pin, and start the servo motor, then the acquisition system started to work. With the balance of mechanical spring on either side of the equipment and the 
mooring rope, the bar slide down with the constant speed controlled by the motor. By means of the stop control unit, the motor will stop when thermocouple reaches the certain position. The experimental system diagram is shown in Fig. 3. When thermocouple sliding down, slide bar pass through a group of optoelectronic switch, the velocity of thermocouple can be obtained.

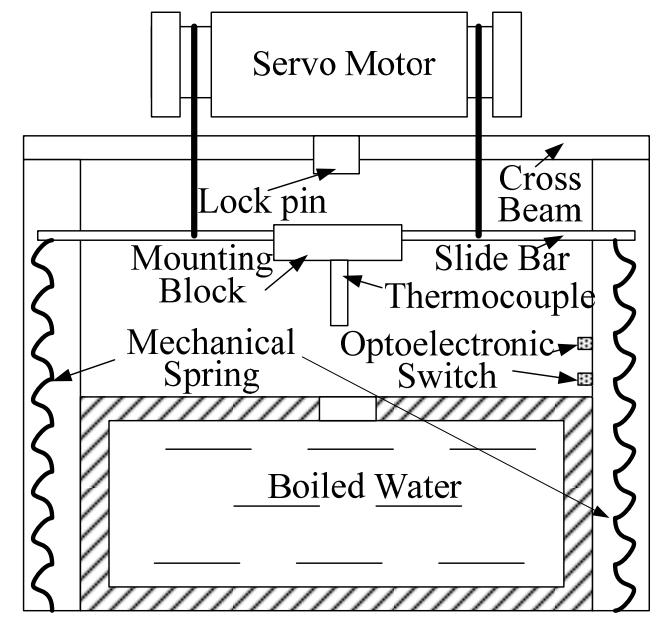

Fig. 2. Water bath calibration system

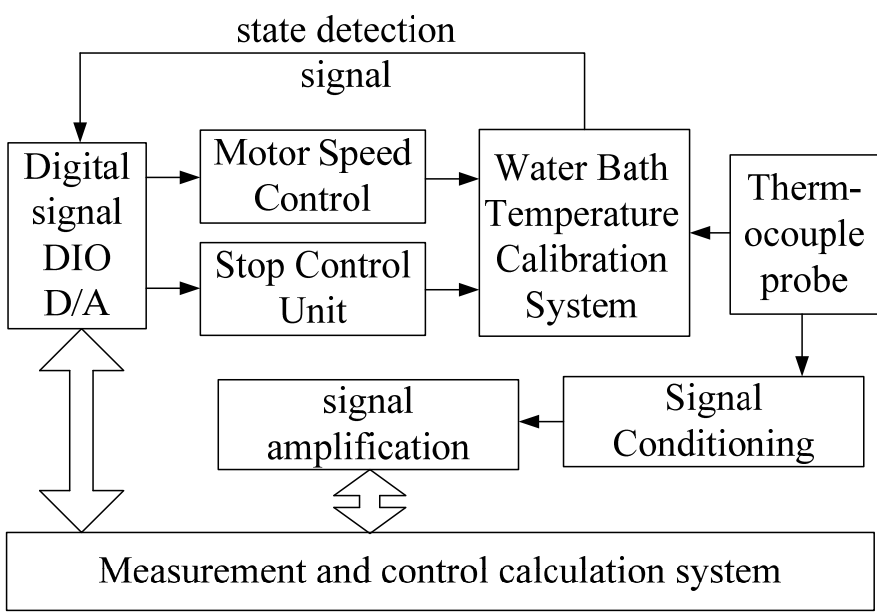

Fig. 3. Block diagram of experimental system

\section{Theory of Measurement}

Neglecting the conduction and radiation heat losses and the effect of catalytic reaction on a thermocouple-wire surface in the heat balance equation of the thermocouple [3], we have the following set of equation for estimating input temperature $\mathrm{T}_{\mathrm{g}}$ :

$$
\tau(\varsigma) \frac{d T}{d t}+T=T_{g}
$$

where the real gas temperature $\mathrm{T}_{\mathrm{g}}$ is the input, $\mathrm{T}$ is output of the thermocouple and $\tau(\zeta)$ is the time constant. In practice, this approach is infeasible as the measured temperature may be noisy. So assuming zero order hold on the inputs and sampling time $\tau_{\mathrm{S}}$, the temperature sensor differential Eq. 1 can be discretized, resulting in the nonlinear difference equation

$$
T_{m}(k)=a(\varsigma) T_{m}(k-1)+b(\varsigma) T_{g}(k-1)
$$

The parameters of the discrete and continuous thermocouple model are related by

$$
a(\varsigma)=\exp \left(-\frac{\tau_{s}}{\tau(\varsigma)}\right) \text { and } b(\varsigma)=1-a(\varsigma)
$$

In general, the thermocouple time constant is defined as follows [4]:

$$
\tau=\frac{\rho c d}{4 h}
$$

where $\rho, \mathrm{c}, \mathrm{d}$ and $\mathrm{h}$ are the density, heat capacity of the thermocouple-wire material, the diameter of the wire, and the local heat transfer coefficient, respectively. From the relationships of heat transfer coefficients between gas and particles for a single sphere [5], the time constant can be described as follows:

$$
\tau=\frac{k}{2+\alpha \cdot u^{\beta}}, k 、 \alpha 、 \beta \text { is constant }
$$

where, $\mathrm{k}$ is decided by physical attributes of the thermocouple, $\alpha, \beta$ are undetermined coefficients.

By means of the nestification of two systems, expect to get the relationships between fluid 
velocity around the thermocouple wire point and the time constant. With the information of the time constant that discussed above, we already get the system function with the parameter unidentified. However the actual circumstance around the thermocouple point may be more complex than the function deduced from the heat transfer theory. In order to get the precise measurement of dynamic system of time constant-fluid velocity, we intend to establish a multiparameter model to describe the system. Generalized total least squares (GTLS), on the other hand, which employ generalized singular value decomposition (GSVD), can produce unbiased parameter estimates [6].

\section{Result and Discussion}

The thermocouple system and time constant-fluid velocity system both excited by signal with a sudden change. We can consider the signals as ideal step for simplicity. So both systems can be modeled as parametric model. By means of the GTLS, we can get the curve of time constant vary with time based on output of the thermocouple, as blue line shown in Fig. 4. We can get that time constant fluctuated more severely at the incipient stage of variation of curve, due to the fact that the system of $\mathrm{G}_{\mathrm{v}-\tau}(\mathrm{u})$ was excited by step velocity single as well. Then we can establish the model of $\mathrm{G}_{\mathrm{v}-\tau}(\mathrm{u})$. Step response of $\mathrm{G}_{\mathrm{v}-\tau}(\mathrm{u})$ is the red line shown in Fig.4. From the figure, the stable value of step response of $\mathrm{G}_{\mathrm{v}-\tau}(\mathrm{u})$ is 0.6 , while the traditional consideration of time constant which is invariable, is just the stable value of regression curve. So it can not reflect the actual situation during the period of time constant fluctuates severely. In addition, if function of time constant is just Eq. 5, the output signal should be step wave when the input signal is step wave either. But the system function is not the case, the reason is that the existing correlation equations of $h$ should not be suitable to high-temperature turbulent flows such as step disturbance. The comparison of time constant curve and step response of $\mathrm{G}_{\mathrm{v}-\tau}(\mathrm{u})$ can be seen in Fig. 4.

Comparison of input reconstruction between variable and invariable time constant is shown in Fig. 5 .

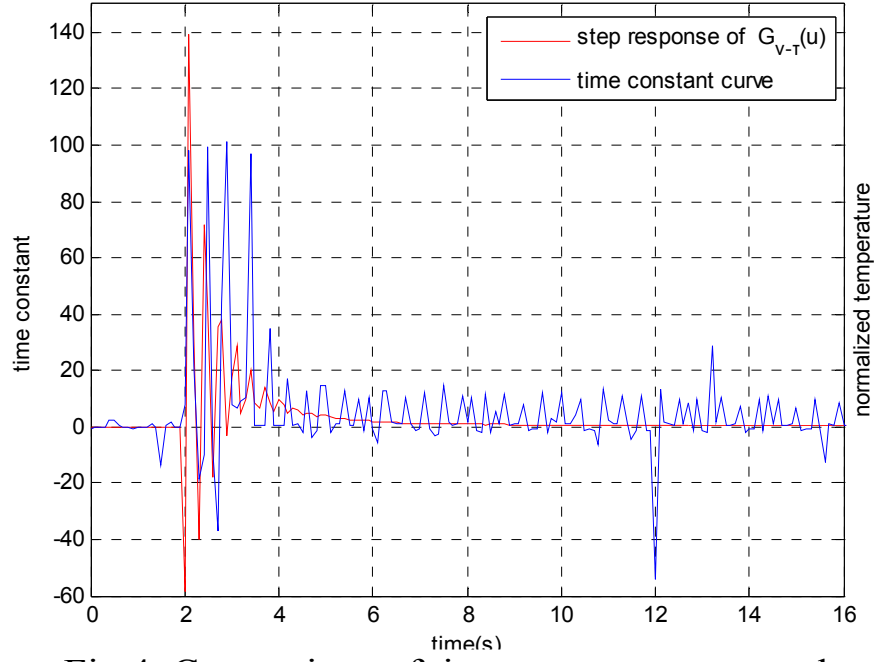

Fig.4. Comparison of time constant curve and step response of $\mathrm{G}_{\mathrm{v}-\tau}(\mathrm{u})$

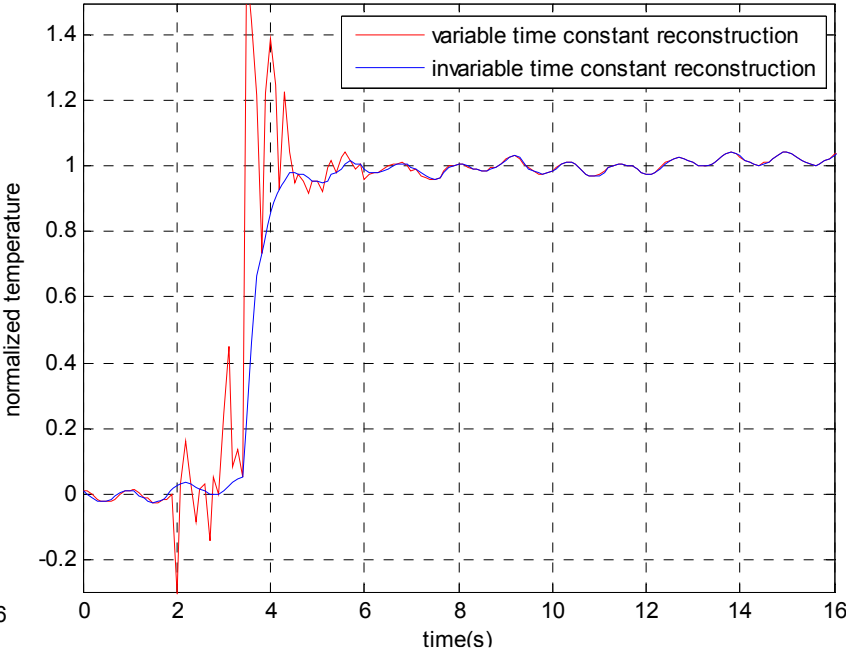

Fig. 5. Comparison of input reconstruction curve between variable and invariable time constant

From Fig. 5, we can get that the rising time (time required rising from $10 \%$ to $90 \%$ of stable value) of reconstructed input signal using variable time constant is $t_{r 1}=0.06 \mathrm{~s}$, in the meantime, the value of which using invariable time constant is $t_{r 2}=1 \mathrm{~s}$. So processing method of variable time constant used in input reconstruction could reflect the excitation signal variation with more wide frequency domain.

\section{Summary}

Novel models and techniques for nonlinear sensor time constant estimation and dynamic sensor 
compensation have been presented. Improved the defect of traditional method of dynamic measurement and calibration of thermocouple which consider time constant is invariable parameter, even the fluid fluctuates more severely. In this paper, we have found out the relationships between time constant of thermocouple and fluid velocity. The variable time constant could reflect the thermal inertia of thermocouple more accurately than a fixed (mean) time constant. Based on this model can provide more accurate method for reconstructing the thermocouple input and therefore compensate the sensor dynamics.

\section{Acknowledgments}

This work is supported by a special key project of National Public Sector of China (Grant No, GYHY200706003) and the National Natural Science Foundation Innovation Group of China (61121003).

\section{References}

[1] Pail G. O'Reilly, Robert J. Kee, Robert Fleck, and Paul T. McEntee: Two-wire thermocouples: A nonlinear state estimation approach to temperature reconstruction, edited by Review of Scientific Instruments. 72, 3449 (2001).

[2] Takawa, M, Y. Ohta: A two-thermocouple probe technique for estimating thermocouple time constants inflows with combustion: In situ parameter identification of a first-order lag system, edited by Review of Scientific Instruments, 69(9), 3370-3378(1998).

[3] Kenneth Kar, Stephen Roberts, Richard Stone, Martin Oldfield, Boyd French: Instantaneous Exhaust Temperature Measurements Using Thermocouple Compensation Techniques, edited by SAE Transactions, Paper No. 2004-01-1418(2004).

[4] L. J. Forney and G. C. Fralick: Two wire thermocouple: Frequency response in constant flow, edited by Review of Scientific Instruments, 65, 3252 (1994).

[5] Tomohiro Akiyama, Reijiro Takahashi, Jun-ichiro Yagi, ISIJ International, Vol. 33 (1993).

[6] P. C. Hung, G. Irwin, R. Kee, and S. McLoone: Difference equation approach to two-thermocouple sensor characterization in constant velocity flow environments, edited by Review of Scientific Instruments. 76, 024902 (2005); 